\title{
July 2019 Critical Care Case of The Month: An 18-Year-Old with Presumed Sepsis and Progressive Multisystem Organ Failure
}

\author{
Robert A. Raschke, MD \\ The University of Arizona College of Medicine - Phoenix \\ Phoenix, AZ USA
}

\section{History of Present IIIness}

An 18-year-old female student from Flagstaff was transferred to our hospital for refractory sepsis. She had presented with a 2 week history of fever, malaise, sore throat, myalgias, arthralgias and a rash.

\section{$\mathrm{PMH}, \mathrm{SH}$ and $\mathrm{FH}$}

She reported no significant past medical history or family history. She attended cosmetology school, denied smoking or drug abuse and was sexually monogamous. She had only traveled in-state, did not hike or camp and her only animal exposure was playing with her two pet Great Danes.

\section{Physical Examination}

The patient had a fever of $38.5^{\circ} \mathrm{C}$. on original presentation. HEENT exam was reported as unrevealing. Lungs were clear. There were no heart murmurs and the abdominal exam was unremarkable. No joint effusions were apparent. A rash was mentioned, but not described and it apparently disappeared shortly after admission.

Initial laboratory testing was significant for WBCC of $12.1 \mathrm{~K} / \mathrm{mm} 3$, creatinine of 1.5 $\mathrm{mg} / \mathrm{dL}$ and $\mathrm{AST}$ of $45 \mathrm{IU} / \mathrm{L}$. A rapid influenza screen, urinalysis and chest radiography were unrevealing. Blood cultures were drawn and intravenous fluids, piperacillin/tazobactam and azithromycin were administered. Over the next four days, the fever persisted and the blood cultures resulted in no growth. Serial laboratory values demonstrated progressive worsening in renal function and increasing hepatic enzymes. The patient became dyspneic and developed rales and progressive hypoxia prompting transfer.

On arrival in our ICU, the patient was alert, in mild respiratory distress and hypotensive to $78 / 43 \mathrm{mmHg}$, requiring immediate initiation of intravenous norepinephrine. She reported nausea and severe diffuse myalgia and arthralgia. On examination, she was illappearing with blood pressure 101/58 (on norepinephrine at $25 \mathrm{mcg} / \mathrm{min}$ ), heart rate 104 beats $/ \mathrm{min}$, respiratory rate 33 breaths $/ \mathrm{min}$, temperature $38.8^{\circ} \mathrm{C}$. She had mild oropharyngeal erythema, some shotty cervical lymph nodes, bilateral rales, mild epigastric and right upper quadrant tenderness, and a macular erythematous rash approximately $14 \times 29 \mathrm{~cm}$ on her left forearm that disappeared within several hours.

Her ICU admission chest x-ray is shown in Figure 1. 
Figure 1. Admission ICU portable chest X-ray showing bilateral areas of consolidation.

Her laboratory evaluation showed the following:

- WBCC: $2,500 / \mathrm{mm}^{3} 63 \%$ segs with toxic granulation/vacuolated segs

- Hemoglobin/Hematocrit: $7.9 \mathrm{~g} / \mathrm{dL} / 26.7 \%$

- Platelets: $50,000 / \mathrm{mm}^{3}$

- BUN/creatinine: $23 / 1.25 \mathrm{mg} / \mathrm{dL}$

- AST/ALT: 246/189 IU/L (normal 10-40 and 7-56)

- PT: $20.9 \mathrm{sec}$

- Lactate: $4.5 \mathrm{mmol} / \mathrm{L}$

- Urinalysis: bland sediment, without bacteria or leukocytes

- $\quad$ ABG: 7.33, pCO2 34, pO2 78 (on 45\% FiO2 by ventimask)

- Transthoracic echocardiogram showed normal LV and RV size and systolic function with no vegetations

- US abdomen showed hepatosplenomegaly, retroperitoneal lymphadenopathy, and normal kidneys and ureters.

What are diagnostic considerations at this time?

1. Rocky mountain spotted fever (RMSF)

2. Acute retroviral syndrome

3. Still's disease

4. Systemic lupus erythematosus (SLE)

5. All of the above 


\section{Correct! \\ 5. All the above}

Rocky mountain spotted fever (RMSF) is endemic in eastern Arizona, and is a tickborne disease characterized by an acute febrile illness that can result in multisystem organ failure. $90 \%$ of cases manifest a petechial macular/popular rash with predilection for the palms and soles. Our patient did not visit parts of the state where RMSF has been reported, did not have recognizable risk factors for tick bite and did not have the typical rash of RMSF, however empirical doxycycline was started pending results of RMSF (which were negative). Most patients are asymptomatic at the onset of HIV infection, but a minority manifest the acute retroviral syndrome (ARS), with fever, lymphadenopathy, sore throat, myalgia, arthralgia and rash - typically small erythematous macules on the face and upper trunk. ARS has rarely been associated with pneumonitis, rhabdomyolysis and opportunistic infections that occur during the transient CD4 lymphopenia that can occur years before the patient would be expected to manifest AIDS.

Still's disease is a seronegative rheumatic disease characterized by quotidian (daily) fevers, arthralgia, myalgia, splenomegaly and an evanescent "salmon-colored" macular rash similar to the one we observed in our patient. Still's disease does not cause multisystem organ failure unless it triggers secondary hemophagocytic lymphohistiocytosis (HLH) or macrophage activation syndrome as some have called it. Systemic lupus erythematosus can present with the same set of constitutional symptoms, lymphadenopathy, splenomegaly and multisystem organ failure including glomerulonephritis, pneumonitis, and pancytopenia. Multiple rashes can be seen including malar, discoid, photosensitivity and palpable purpura (in patients with cutaneous vasculitis associated with SLE)

Antibiotics were broadened to meropenem, vancomycin and doxycycline. HIV, EBV, CMV, hepatitis serology, ANA and rheumatoid factor were ordered.

Which laboratory tests is most likely to suggest a specific diagnosis?

1. Anti-nuclear antibody (ANA)

2. Erythrocyte sedimentation rate (ESR)

3. Ferritin

4. Procalcitonin

5. Rheumatoid factor 


\section{Correct!}

\section{Ferritin}

The antinuclear antibody (ANA) is a nonspecific reactant seen in a variety of diseases. Similarly, the erythrocyte sedimentation rate (ESR) is a non-specific acute phase reactant only occasionally valuable in making a specific diagnosis - for instance, it can be useful in patients with a high clinical pretest probability for the diagnosis of temporal arteritis. Procalcitonin is released by a wide variety of human cells in response to systemic bacterial infection. Although initially promising as a tool to identify bacterial sepsis, it lacks sensitivity and specificity and should generally not be used to make decisions about whether empirical antibiotics should be started. There is some evidence that it might be helpful in discontinuing antibiotics in patients with community acquired pneumonia, but the value of that practice has recently been called into question. Rheumatoid factors are antibodies against the Fc portion of IgG. They are non-sensitive and non-specific for rheumatoid arthritis and likely are used mostly because of historical overestimation of their clinical value. Our patient's presentation was not compatible with RA and she did not have arthritis on physical examination. RF is typically negative in the majority of patients with Stills.

Ferritin is also a non-specific acute phase reactant, but it is typically strikingly elevated in cases of Still's disease or HLH. These diagnoses are much less likely if ferritin is $<500 \mathrm{ng} / \mathrm{mL}$, and clinical suspicion is increased if ferritin is very elevated (i.e. $>2000$ $\mathrm{ng} / \mathrm{mL}$ ). Other easily-obtained laboratory findings of $\mathrm{HLH}$ include hypofibrinogenemia and hypertriglyceridemia.

Further lab testing of our patient revealed:

- Fibrinogen: $417 \mathrm{mg} / \mathrm{dL}$ (normal150-400)

- Triglycerides: $456 \mathrm{mg} / \mathrm{dL}$

- Ferritin: $14,281 \mathrm{ng} / \mathrm{mL}$ (normal 12-300)

The patient is presenting with presumed sepsis unresponsive to broad spectrum antibiotic therapy, with persistent fever, progressive multisystem organ failure, bicytopenia and severe hyperferritinemia. This syndrome is most compatible with the diagnosis of secondary $\mathrm{HLH}$.

What should be done next?

1. A bone marrow biopsy

2. Measure soluble interleukin-2 receptor and NK cell activity

3. Start empirical corticosteroids before the diagnosis is confirmed

4. 1 and 2

5. All of the above 


\section{Correct! \\ 5. All of the above}

The diagnosis of HLH requires at least 5 of 8 criteria "HLA-2004" criteria are met (1). However, completing the diagnostic work-up is time-consuming. A bone marrow biopsy is often delayed unless the intensivist performs it themselves. sIL2-R and NK cell activity tests are send-outs that often take 1-2 weeks to result. Patients in shock and multi-system organ failure from secondary $\mathrm{HLH}$ are at high mortality risk, and empirical immunotherapy should be started if clinical suspicion is high, as in this case, while the diagnosis is being confirmed.

Intravenous methylprednisolone, 60mg every six hours was started. A bone biopsy was performed by the intensivist. A marrow smear from the patient is shown in Figure 2. The bone marrow was infiltrated by large numbers of histiocytes, many of which were ingesting hematopoetic cells normally residing in the bone marrow.

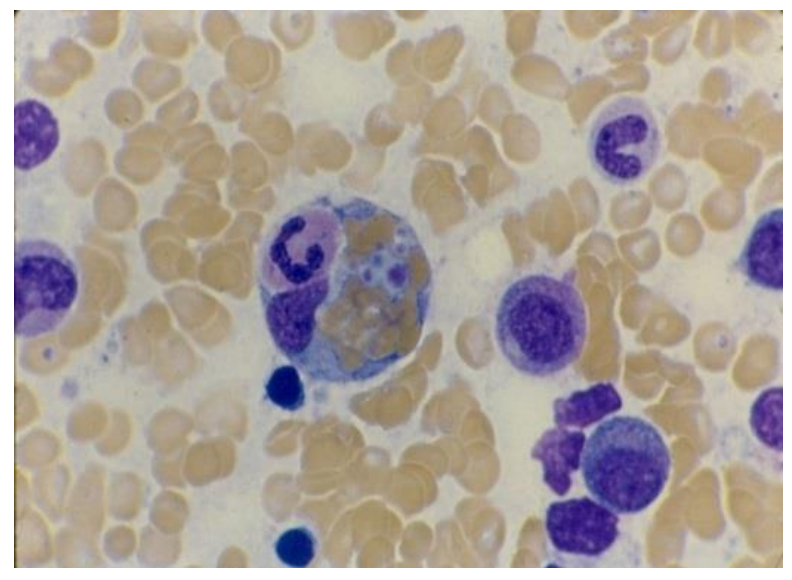

Figure 2. Phagocytosis of a neutrophil, platelets and red blood cells by a single histiocyte (a tissue-residing macrophage).

Soluble interleukin-2 receptor was $6265 \mathrm{u} / \mathrm{mL}$ (normal 241-846) and natural killer cell activity was undetectable. An abdominal ultrasound showed mild hepatosplenomegaly.

What is the patient's diagnosis?

1. Adult Still's Disease

2. Macrophage activation syndrome

3. Primary HLH

4. Secondary HLH

5. Possibly all the above 


\section{Correct! \\ 5. Possibly all the above}

$\mathrm{HLH}$ is a highly fatal disease caused by dysregulation of the immune system, severe hypercytokinemia and resultant multiorgan failure. Primary $\mathrm{HLH}$ is a genetic disorder of impaired NK cell function presenting early in childhood, but most cases of HLH in adults do not undergo testing for genetic abnormalities and are presumed to be secondary, although in some cases, adults with $\mathrm{HLH}$ have been found to have inborn errors. The patient presented above was later tested for known genetic abnormalities associated with primary HLH and none were found. Secondary HLH can be triggered by a wide variety of infections (particularly EBV and herpes virus infections), malignancies and rheumatological diseases (particularly Still's disease) (2). Some authors believe that HLH secondary to Still's is a distinct syndrome and have used the term "macrophage activation syndrome" to describe it. But MAS and secondary HLH share many clinical features, diagnostic criteria and treatment options.

The patient meets all eight "HLH 2004" diagnostic criteria for hemophagocytic lymphohistiocytosis $(\mathrm{HLH})$ - but only five are required to confirm the diagnosis (Table 1) (1).

Table 1. HLH 2004 diagnostic criteria for HLH

- Fever

- Hyperferritinemia

- Splenomegaly

- Bi or pancytopenia

- Hypofibrinogenemia/hypertriglyceridemia

- Histiocytic hemophagocytosis on tissue biopsy

- Elevated soluble interleukin 2 receptor

- Severely decreased NK cell activity

Histological evidence of $\mathrm{HLH}$ on tissue biospy is neither necessary or sufficient to make the diagnosis (3). Hyperferritinemia is also non-specific for $\mathrm{HLH}$, but is present in nearly all cases.

Secondary HLH is likely underdiagnosed in the adult ICU. We have previously shown that HLH can present as sepsis/septic shock refractory to antibiotic therapy (4). In an autopsy study, Straus et al. (5) identified HLH in the bone marrow of 35 of 107 patients who died from sepsis. HLH was identified in 9/25 (36\%) of critically ill patients with confirmed influenza $A(\mathrm{H} 1 \mathrm{~N} 1)$ infection (6). We have personally diagnosed 27 patients with secondary HLH in our ICU, and have observed that it is most often originally misdiagnosed as typical sepsis or septic shock. The diagnosis of HLH is usually suspected only when the patient continues to deteriorate despite broad spectrum antibiotics and progressive multisystem organ failure and bone marrow failure appear to be imminently life-threatening. In many cases the development of pancytopenia triggered suspicion of the diagnosis, which was then supported by finding hyperferritinemia. Treatment has almost always been started before sIL-2r and NK cell 
activity testing is resulted. The mortality of this syndrome presenting in the adult ICU exceeds $50 \%$ even when the diagnosis is recognized and rapidly treated.

Which of the following have been used to treat $H L H$ ?

1. Anakinra

2. Corticosteroids

3. Etoposide

4. Methotrexate

5. All of the above 


\section{Correct! \\ 5. All of the above}

In many cases of $\mathrm{HLH}$, an underlying infection or malignancy requires specific therapy. Non-specific immunotherapy of HLH typically involves the use of corticosteroids, intravenous immunoglobulin G (IVIg) and etoposide (7). However, etoposide, a myelosuppressive chemotherapy agent, often causes severe pancytopenia in critically ill patients with hepatic and/or renal dysfunction typical of sepsis (7).

Anakinra $($ Kineret $\AA)$ is a recombinant modified human interleukin 1 receptor antagonist used to treat rheumatoid arthritis. Increasing evidence suggests efficacy and safety of anakinra in both children and adults with HLH (8). In this issue of SWJPCC, Henry and Raschke (9) add to the mounting observational evidence of the efficacy of anakinra in adults reporting a case series of 7 adult patients with $71 \%$ survival.

The patient described above was treated with corticosteroids, IVIg and anakinra and gradually recovered from her illness over a period of months.

\section{References}

1. Henter J, Horne A, Aricó M, et al. HLH-2004: Diagnostic and therapeutic guidelines for hemophagocytic lymphohistiocytosis. Pediatr Blood Cancer. 2007;48:124-31. [CrossRef] [PubMed]

2. Hayden A, Lin M, Park S, Pudek M, Schneider M, Jordan MB, Mattman A, Chen LYC. Soluble interleukin-2 receptor is a sensitive diagnostic test in adult HLH. Blood Adv. 2017 Dec 6;1(26):2529-34. [CrossRef] [PubMed]

3. Stéphan F, Thiolière B, Verdy E, Tulliez M. Role of hemophagocytic histiocytosis in the etiology of thrombocytopenia in patients with sepsis syndrome or septic shock. Clin Infect Dis. 1997 Nov;25(5):1159-64. [CrossRef] [PubMed]

4. Raschke RA, Garcia-Orr R. Hemophagocytic lymphohistiocytosis: A potentially underrecognized association with systemic inflammatory response syndrome, severe sepsis, and septic shock in adults. Chest 2011;140:933-8. [CrossRef] [PubMed]

5. Strauss R, Neureiter D, Westenburger B, Wehler M, Kirchner T, Hahn EG. Multifactorial risk analysis of bone marrow histiocytic hyperplasia with hemophagocytosis in critically ill medical patients--a postmortem clinicopathologic analysis. Crit Care Med. 2004 Jun;32(6):1316-21. [CrossRef] [PubMed]

6. Beutel G, Wiesner O, Eder M, et al. Virus-associated hemophagocytic syndrome as a major contributor to death in patients with 2009 influenza A (H1N1) infection. Crit Care. 2011;15(2):R80. [CrossRef] [PubMed]

7. Wohlfarth $\mathrm{P}$, Agis $\mathrm{H}$, Gualdoni GA, Weber J, Staudinger T, Schellongowski P, Robak O. Interleukin 1 receptor antagonist anakinra, intravenous immunoglobulin, and corticosteroids in the management of critically ill adult patients with hemophagocytic lymphohistiocytosis. J Intensive Care Med. 2017 Jan 1:885066617711386. [CrossRef] [PubMed] 
8. Rajesekaran S, Kruse K, Kovey K, et al. Therapeutic role of anakinra, an interleukin1 receptor antagonist, in the management of secondary hemophagocytic lymphohistiocytosis/sepsis/multiple organ dysfunction/macrophage activating syndrome in critically ill children. Pediatr Crit Care Med. 2014;15:401-8. [CrossRef] [PubMed]

9. Henry K, Raschke RA. An observational study demonstrating possible efficacy of interleukin-1 antagonist (anakinra) in critically-ill patients with hemophagocytic lymphohistiocytosis. Southwest J Pulm Crit Care. 2019;18(6):177-86. [CrossRef] 